\title{
The Transcriptional Response of Arabidopsis thaliana L. Genes AtKu70, AtRAD51 and AtRad1 to X-Ray Radiation
}

\author{
Sergey Litvinov and Namik Rashydov \\ Institute of Cell Biology and Genetic Engineering, National Academy of Sciences of Ukraine, 148 Zabolotnogo, Kiev-03143, Ukraine
}

\begin{abstract}
The study of influence of the fractionated and acute ionizing radiation on plants revealed that it is able to induce genomic instability. The hypothesis that transcription rate of several evolutionary conserved DNA repair genes AtKu70, AtRAD51 and AtRad1, which keeps genome stability in cells of model plant Arabidopsis thaliana, changes differently depending on dose and mode of ionizing radiation exposure had been tested. Gel electrophoresis-based reverse transcription polymerase chain reaction (RT-PCR) method was used for quantifying mRNA transcription levels. The data demonstrated that mode and dose of irradiation affect transcription rate of the genes AtKu70, AtRAD51 and AtRad1. The fractionated and acute X-ray irradiation may result in adaptive response through the induction of key DNA double-strand break (DSB) repair genes AtKu70 and AtRAD51, as well as in genome instability through transcriptional activation of error-prone AtRad1-mediated DNA DSB repair combined with decreased expression of AtRAD51. In plants at doses within the range of 3-9 Gy, an adaptive influence is prevailed, but at doses of 12-21 Gy an error-prone repair of double-strand DNA damage is activated. Fractionation of dose has a significant effect on the transcription of the genes AtKu70, AtRAD51 and AtRad1 only at doses of 15 Gy and 21 Gy. Acute dose of 15 Gy activates error-prone AtRad1-mediated DSB repair and repressed both AtRAD51-dependent and AtKu70-dependent repair pathways, while fractionated irradiation at the same total dose induces more accurate homologous recombination and canonical non-homologous end joining of the DNA strands. In case of A. thaliana exposed to X-rays at dose $21 \mathrm{~Gy}$, the situation is going reversed because of strong induction of the all three genome caretaker genes in leaves of acutely irradiated plants in contrast to the plants under fractionated exposure.
\end{abstract}

Key words: X-ray exposure, DNA repair, gene expression, transcription rate, genome instability.

\section{Introduction}

Preserving the integrity and self-identity of the genetic apparatus is tremendously important for both developing and mature organisms. It is acquiring special significance for the higher plant [1]. The sedentary plants are more susceptible to the action of the adverse genotoxic factors in comparison with actively moving animals. Restricted ability of the cells of post-embryonic tissues to repopulation and limited mitotic potential of the meristematic cells taken together probably resulted in progressive evolution of the effective repair of the lethal DNA damages in plants $[1,2]$. Perhaps this explains radioresistance of seeds of the most of the higher plants. However, the

Corresponding author: Namik Rashydov, Ph.D., research fields: radiobiology, biophysics, plant physiology, molecular biology. sensitivity of plants to the small doses of ionizing radiation, especially fractionated or chronic, is little different from the radiosensitivity of animal organisms [3]. The reason for these similarities and differences in the biological action of radiation on plant and animal objects remains unclear, but presumably is associated with DNA repair effectiveness [4].

According to modern concepts, basic proteins and enzymatic pathways for repair of the main radiation-induced lesions-DNA breaks are evolutionary conserved among eukaryotes. Therefore, it can be assumed that the particular modification of radiosensitivity of plant cells under the influence of radiation might be caused by the change in the transcriptional activity of genome caretaker genes in a manner resembling those found in mammalian cells [5-7]. 
The main pathways for genome recovery after exogenous and endogenous DNA damage include (directly or as the one of the intermediate stages of processing of damaged DNA) repair of double-stranded and single-stranded DNA breaks with non-ligatable ends, gap filling and DNA interstrand crosslink resolution. As known, three enzymes play a key role in mentioned above repair pathways for maintaining the structural integrity of plant DNA, i.e., RAD51, a homologue of bacterial recombinase RecA; Ku70, a component of DNA-protein kinase complex and Rad1 (UVH1), endonuclease/exonuclease involved in the removal of damaged nucleotides and "nicked" ssDNA ends by Rad1/Rad10 (XPF/ERCC1) complex. Genes coding these proteins belong to the important group of caretaker genes. The effect of the acute and fractionated X-ray ionizing irradiation of the mature Arabidopsis thaliana plants at doses significantly smaller than median lethal dose $(\sim 150$ Gy) on the expression of the genes AtKu70, AtRAD51 and AtRad1 can touch not only repair of induced single-strand DNA breaks (SSBs) and double-strand DNA breaks (DSBs), but also effectiveness of preventing of endogenous DNA damage.

The aim of this investigation was to examine dose-effect dependence of transcriptional response of caretaker genes AtKu70, AtRAD51 and AtRad1 under acute and fractionated X-ray irradiation at non-lethal doses. As a model for repeated action of radiation, a fractionation of total dose into three equal fractions had been served, with one fraction per $24 \mathrm{~h}$ (the sum of portions was equal to the each acute dose), at which mode the cells have repaired the majority of reparable primary-induced DNA damage before exposure to the next fraction of irradiation. As a reference gene, AtEf1a have been used, one of the housekeeping genes, which encodes an isoform of the alpha subunit of the elongation factor- 1 complex, which is responsible for the enzymatic delivery of aminoacyl tRNAs to the ribosome. Strong correlation between transcription rate of this gene and general transcription level is common to the viable plant cells.

\section{Materials and Methods}

\subsection{X-Ray Exposure}

The relative concentration of the pre-mRNAs of genes AtKu70, AtRAD51 and AtRad1 in rosette leaves of A. thaliana Col-0 plants was examined after acute and fractionated irradiation in a RUM-17 X-ray equipment (National Cancer Institute, Kiev, Ukraine, 2015). Photon energies of $180 \mathrm{keV}$ had been used in the experiments as a model for low linear energy transfer (LET) ionizing radiation exposure. Dose rate was $89 \mathrm{cGy} / \mathrm{min}$ and the summarizing dose ranged from $3 \mathrm{~Gy}$ to $21 \mathrm{~Gy}$. When dose had been fractioned, the time interval between fractions was $24 \mathrm{~h}$. Dosage and exposure modes were as follows: 3 Gy applied as acute versus three fractions $1 \mathrm{~Gy}+1 \mathrm{~Gy}+1 \mathrm{~Gy}$ as fractioned dose with interval $24 \mathrm{~h}$; similarly, 6 Gy vs. $2 \mathrm{~Gy}+2 \mathrm{~Gy}+2 \mathrm{~Gy}, 9$ Gy vs. $3 \mathrm{~Gy}+3 \mathrm{~Gy}+3 \mathrm{~Gy}, 12$ Gy vs. 4 Gy +4 Gy +4 Gy, 15 Gy vs. 5 Gy +5 Gy + 5 Gy and 21 Gy vs. 7 Gy +7 Gy +7 Gy. The doses had chosen much lower than the median lethal dose $\mathrm{LD}_{50}$, but nevertheless effective in induction of phenotypic effects, such as inhibition/stimulation of growth processes and flowering. Plants were germinated on soil in open growth-chambers at temperature $20-24{ }^{\circ} \mathrm{C}$ under standard conditions of $16 / 8 \mathrm{~h} \mathrm{light/dark}$ per day, where the light mode of illumination was on $80 \mu \mathrm{M} / \mathrm{m}^{2} \cdot \mathrm{s}$. Plants were exposed to X-rays at $35 \mathrm{~d}$ of growth before beginning of flowering. Experiments had been repeated for three times.

\subsection{RNA Extraction and Reverse Transcription}

Reverse transcription polymerase chain reaction (RT-PCR) was performed for detection of differences in gene's transcription rate [8]. On the template of mRNA extracted from fresh leaves $2 \mathrm{~h}$ after irradiation (standard time for early transcriptional response detection), a reverse transcription reaction 
was carried out and then PCR has been performed. Total RNA was isolated from $500 \mathrm{mg}$ tissue of 2-3 rosette leaves from each of 25 plants per dose value variant using the RIBO-Sorb kit from AmpliSens (Russian Federation). The procedure involved disruption and mechanical homogenization of fresh tissue material frozen with liquid nitrogen just before RNA isolation. Reverse transcription of total RNA for the preparation of cDNA was done using REVERTA-L kit from AmpliSens (Russian Federation) containing M-MLV reverse transcriptase and random hexamers as polymerization primers. All manipulations were carried out according to the manufacturer's protocol.

\subsection{PCR Conditions}

PCR (GeneAmp PCR System 2400, Applied Biosystems, USA) was performed in $20 \mu \mathrm{L}$ total volume, using $5 \mu \mathrm{L}$ cDNA as a template, $9.8 \mu \mathrm{L} \mathrm{ddH}_{2} \mathrm{O}$, $2 \mu \mathrm{L}$ of $10 \mathrm{mM}$ dNTP mix (Thermo Fisher Scientific, USA Fermentas), $0.2 \mu \mathrm{L}$ Taq DNA polymerase (Thermo Fisher Scientific, USA), $2 \mu \mathrm{L}$ of Taq DNA polymerase buffer, $0.5 \mu \mathrm{L}$ of $10 \mu \mathrm{g} / \mathrm{L}$ each of two primers, forward and reverse. The PCR conditions were as follows: heating to $95{ }^{\circ} \mathrm{C}$ for $4 \mathrm{~min} ; 35$ cycles of $95{ }^{\circ} \mathrm{C}$ for $45 \mathrm{~s}, 63{ }^{\circ} \mathrm{C}$ for $45 \mathrm{~s}$ and $72{ }^{\circ} \mathrm{C}$ for $60 \mathrm{~s}$, followed by final extension for $10 \mathrm{~min}$. Target cDNA sequences were amplified with oligonucleotide primers: Fw 5'-TCCGTCCGGCTATTCCTGGC-3', Rev 5'-TGCGTCACCGGCTTTTCGCT-3' for At1g16970 (AtKu70); Fw 5'-ACCCAGCACGGACCTTTCCC-3', Rev 5'-ATCGAGCTTCCGCTTCTGGC-3' for At5g20850 (AtRAD51); $\mathrm{Fw}$ 5'-AAGCCAATGCTGTTCGCCCC-3', Rev 5'-TCTTCCGCCTGCTTTTCGGG-3' for At5g41150 (AtRad1); Fw 5'-AGCCCCTTCGTCTCCCACTT-3', Rev 5'-GCCAAGTACCTCCGCCACCT-3' for At1g07940 (AtEf1a). The PCR with the product of reverse transcription without M-MLV reverse transcriptase as negative control was used, for disabling false positives due to the occasional genomic DNA contamination.

\subsection{Gel Densitometry and Transcription Rate Evaluation}

Differences in the relative level of transcription were examined using densitometric quantification of $1 \%$ agarose and $0.005 \%$ of $10 \mathrm{mg} / \mathrm{L} \mathrm{EtBr}$ containing gel electrophoresis images. Images were captured with a Cannon PowerShot G5 camera and then ImageJ program (National Institutes of Health, USA) was used to measure band intensities. The relative intensities of specific bands were calculated by reference to the 500-bp band from the molecular weight marker (Thermo Fisher Scientific, USA). Results were normalized by the concentration of mRNA of housekeeping gene AtEf1a. An average value of gel images from two repeated PCR reactions per every dose point was analyzed. As a measure of the relative transcription rate of the gene, a normalized concentration of RT-PCR amplification of pre-mRNA (under identical conditions) isolated from the equivalent samples of leaves tissue was taken. The differences in the mature mRNA degradation rate did not affect this estimate.

\subsection{Statistical Analysis}

Obtained data then were averaged using a log-exponential transformation and analyzed using standard statistical methods. Differences between groups were tested for statistical significance using two-tailed Mann-Whitney U test and Kruscal-Wallis test (Monte Carlo method, 10,000 iterations) based on a confidence probability level $P>0.95$. Data presented in graphs and diagrams are averages with sample errors, similar to standard deviations in the case of Gaussian-based statistics, but non-symmetric because of log-exponential transformation of the raw values. The relationship between transcription rates of studied genes was characterized by Spearman's rank correlation and Pearson partial correlation coefficients. 


\section{Results and Discussion}

\subsection{The Contrary Effects of Different Doses of X-Rays on the Activity of DNA Repair Genes}

Dose and transcription rate curves reflect contrary effect of different doses of X-rays on activity of DNA repair genes. As shown in Fig. 1, acute and fractionated irradiation at dose $3 \mathrm{~Gy}$ induce increased expression of the genes AtKu70 and AtRAD51, but transcriptional activity of AtRad1 is not changed. However, transcription rate of a reference gene AtEf1a after acute exposure decreased three folds. Acute irradiation within doses 9-12 Gy has lower stimulative effect on the concentration of AtRAD51 and AtKu70 transcripts up to the absence of stimulation of AtRAD51 or even suppression of transcription of AtKu70 at 12 Gy and 15 Gy. Under condition of acute exposure at dose of $21 \mathrm{~Gy}$, the second maximum of expression level of genes AtKu70, AtRAD51 and AtRad1 is observed. In the case of fractionated exposure, an effect of X-rays is similar at doses lower than $9 \mathrm{~Gy}$, and very differs from acute irradiation influence at doses 12-21 Gy. Fractionated irradiation generally less efficiently induces expression of repair genes AtKu70 and AtRAD51, compared with acute irradiation at stimulating doses $3 \mathrm{~Gy}$ and $21 \mathrm{~Gy}$, but more efficiently induces expression of mentioned genes at inhibitory dose 15 Gy (Fig. 1).

The most intrigued finding is silencing of the gene $K u 70$, the main factor of non-homologous end-joining during DSB repair in eukaryotic somatic cells, when plants were exposed to X-rays at acute dose 12 Gy and also to acute irradiation at $15 \mathrm{~Gy}$. This combines with the activation of Rad1 expression, which involved in highly error-prone single-strand annealing (SSA) and microhomology-mediated end joining (MMEJ) [9-11]. Also, the total expression of AtKu70, AtRAD51 and AtRad1 raised the minimum under conditions of acute radiation exposure at $15 \mathrm{~Gy}$.

Analysis of obtained data confirmed that the most reliable (but in some cases insufficient) marker of radiation-induced transcriptional response is an increased level of the AtRAD51 mRNA. Transcriptional activation of AtRAD51 was observed at doses from $3 \mathrm{~Gy}$ to $9 \mathrm{~Gy}$, and absence of this effect observed at higher doses, except acute dose of $21 \mathrm{~Gy}$ (Fig. 1c and Fig. 2a). It should be mentioned that $R A D 51$ protein takes part not only in DNA repair, but also in meiotic recombination. Therefore, activation of AtRAD51 in floral meristem cells is critical at the reproductive stage of ontogenesis [12].

Interestingly, on the border range of stimulating doses of acute irradiation ( 9 Gy), an additional amplicon of AtKu70 have been detected, the respective mature mRNA $\sim 845$ bp in length (Fig. 1b, $9 \mathrm{~Gy}$, fractionated irradiation) that probably reflect the stimulation of pre-mRNA processing. Also when 15 Gy dose was fractionated on three equal fractions with the $24 \mathrm{~h}$ timeout interval of exposure (the given mode of irradiation stimulates AtKu70 and AtRAD51 expression, but represses AtRad1), an additional unknown AtEF1a amplicon length of $\sim 420$ bp has been detected (Fig. 1a). Further search in the databases of annotated nucleotide sequences gives reason to speculate on RNA interference between the AtEF1a pre-mRNA and complementary siRNA of Ath_wt_00191 (GenBank accession number DQ950747.1). These data indicate possible involvement of mechanisms of mRNA maturation and RNA interference in the regulation of transcriptional response to ionizing radiation.

It was found that the obtained dose and gene transcription rate curves (Figs. 2a and 2b) are in good consistent with the authors' previous observation on radiation-induced modification of growth and development of $A$. thaliana. Thus, $\mathrm{X}$-ray irradiation at doses 3 Gy and 21 Gy stimulates plant stem growth, earlier flowering and pods ripening. Irradiation at dose of 12 Gy has contrarily inhibitory influence on growth and development processes of seedlings. Furthermore, inhibition of stem growth, decrease of the pods size and the average number of pods per plant, increased level 

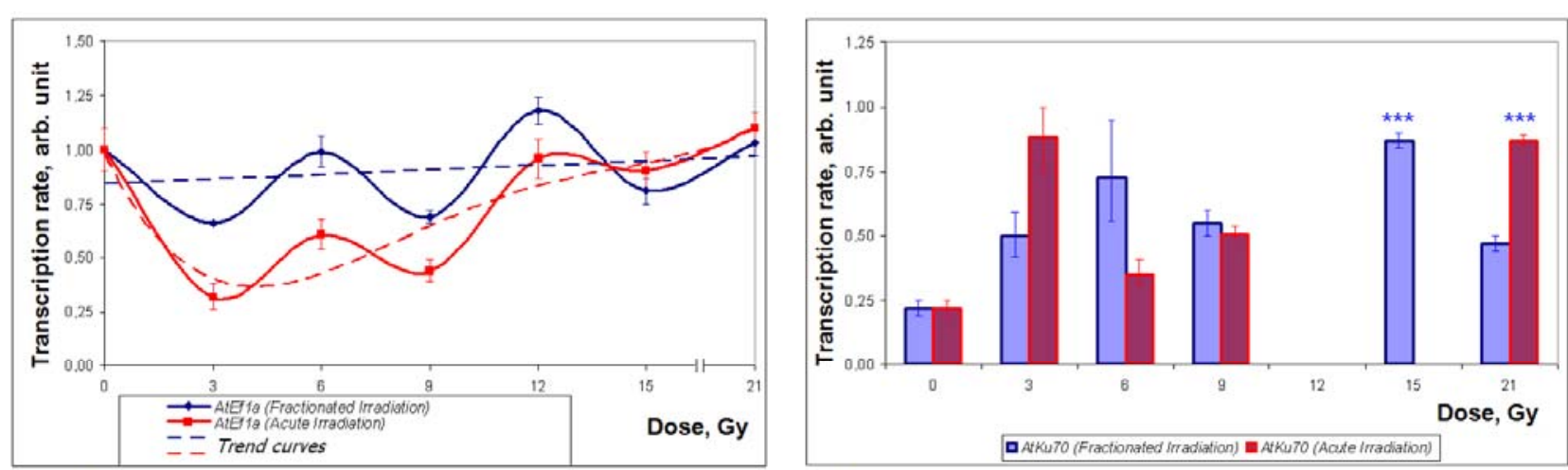

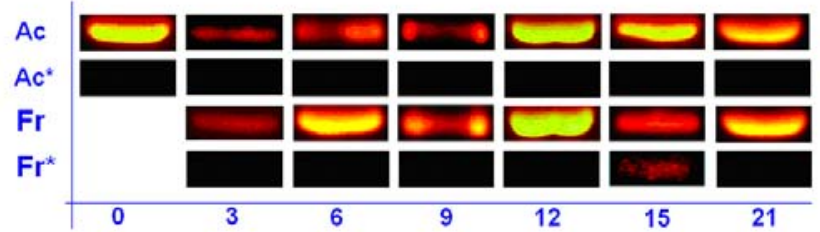

(a) AtEf1a cDNA
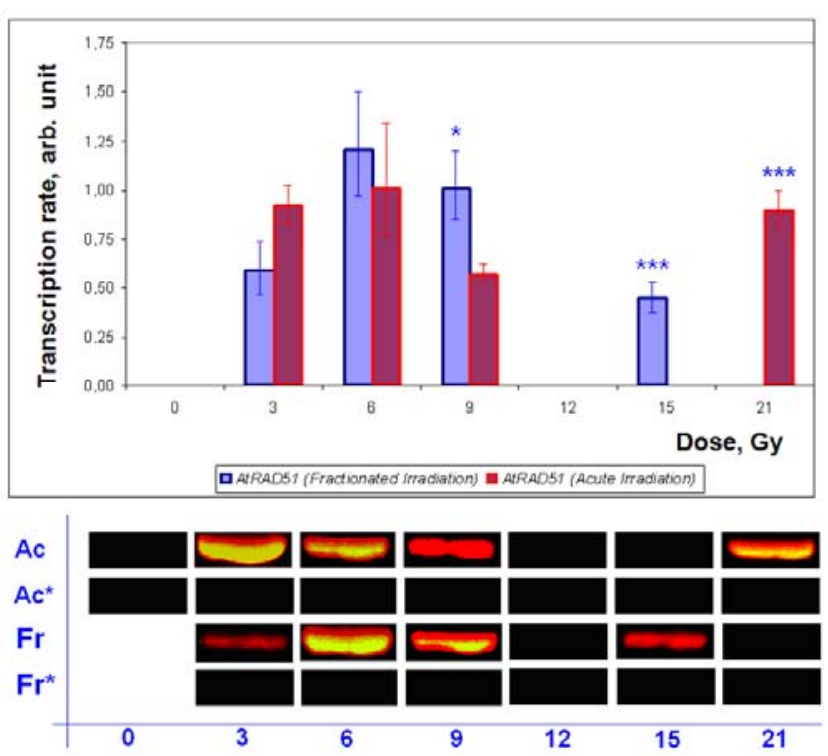

(c) AtRAD51 cDNA

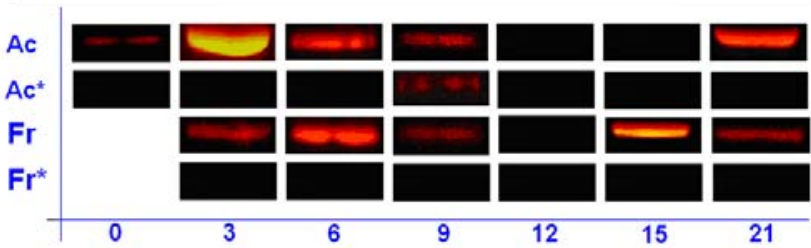

(b) AtKu70 cDNA
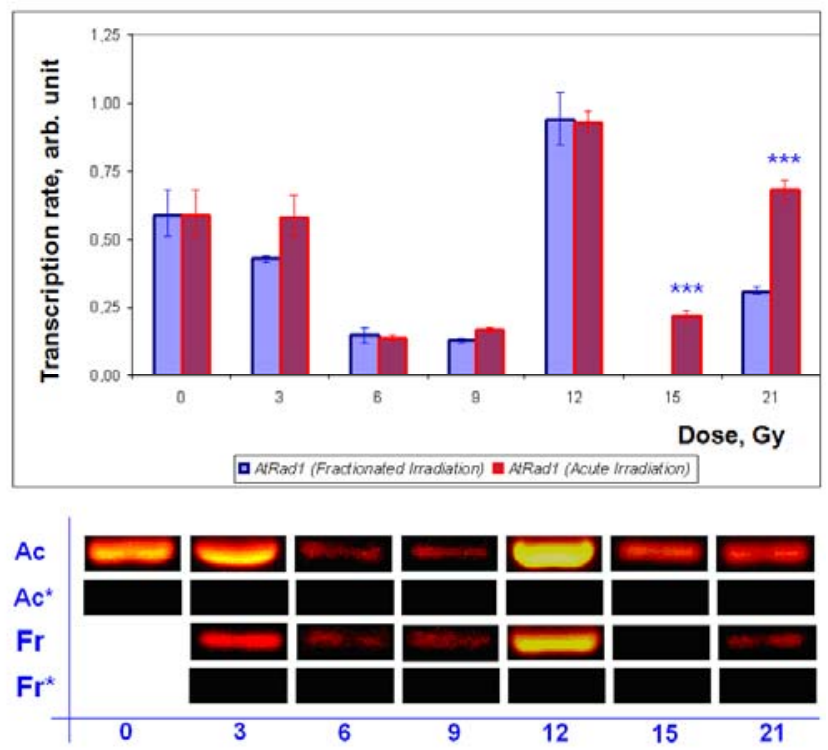

(d) AtRad1 cDNA

Fig. 1 Transcription rate of the genes AtKu70, AtRAD51, AtRad1 under acute and fractionated X-rays exposure.

Ac: acute X-ray irradiation, main amplicon; Ac*: fractionated X-ray irradiation, additional amplicon; Fr: fractionated X-ray irradiation, main amplicon; Fr*: fractionated X-ray irradiation, additional amplicon.

Arbitrary unit is equal to the transcription rate of AtEf1a in leaves of unirradiated plants. The absence of amplicon is an evidence for gene activity, lower than threshold of the densitometric detection of specific mRNA.

Group differences at significance level: * $P \leq 0.05 ; * * P \leq 0.01 ; * * P \leq 0.001$.

of sterility, decreased seed germination, phenotypic abnormalities were observed in A. thaliana grown from seeds of plants, which had been irradiated at dose of $12 \mathrm{~Gy}$. These markers indicate genome instability in meristematic cells and gametes, as well as in embryo tissues cells in $F_{1}$ generation progeny of irradiated plants.

Significantly, the doses of X-ray, which led to stimulation of vegetative and generative sphere of plant vital activity, coincide with doses inducing or increasing transcription rate of genes AtRAD51 and AtKu70. Contrariwise, exposure to ionizing radiation 


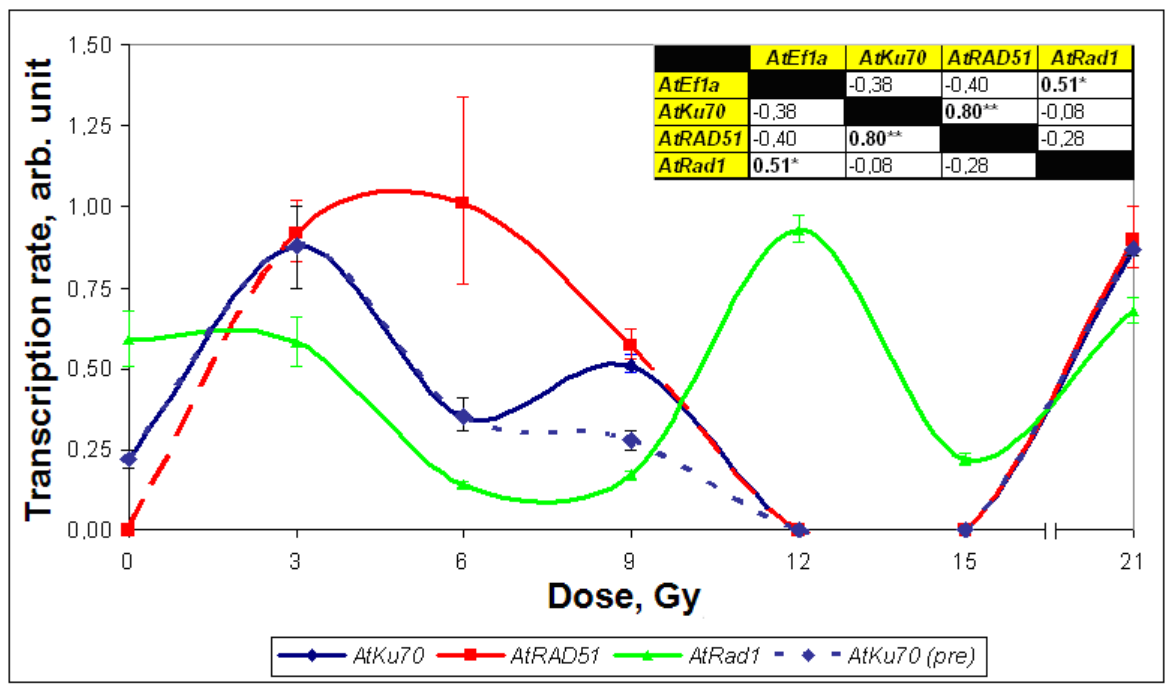

(a) Acute irradiation

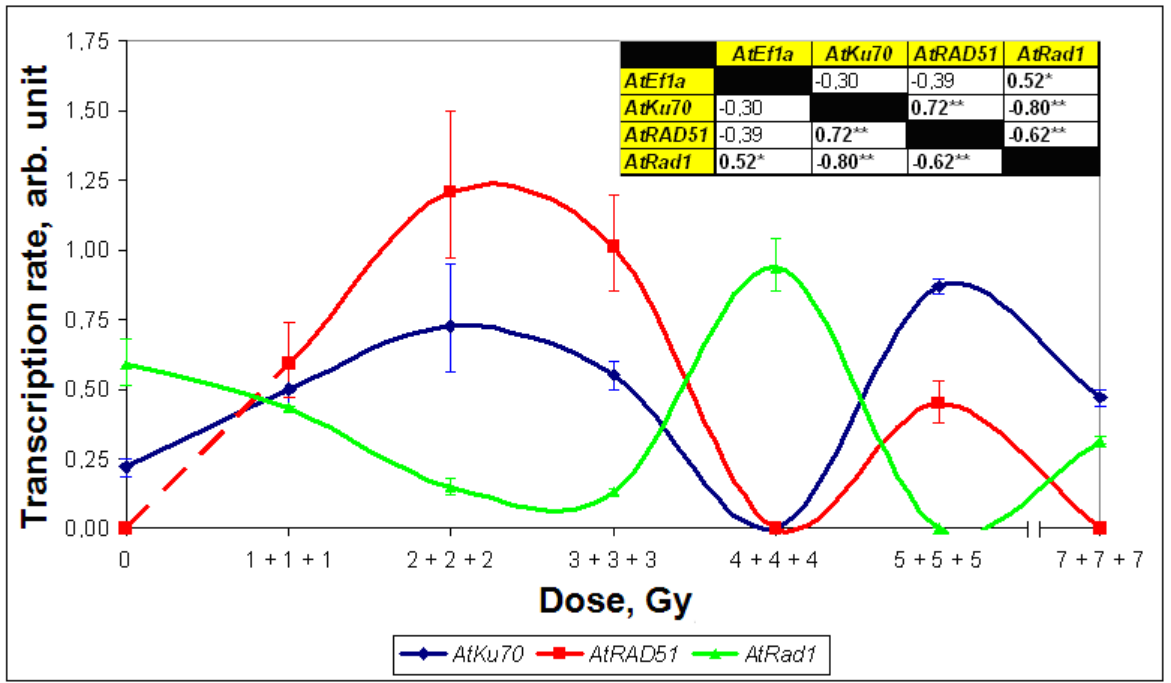

(b) Fractionated irradiation

Fig. 2 Relative transcription rate of genes AtKu70, AtRAD51, AtRad1 under different doses of X-rays exposure.

Arbitrary unit is equal to the transcription rate of AtEf1a in leaves of unirradiated plants. The absence of amplicon is an evidence for gene activity, lower than threshold of the densitometric detection of specific mRNA.

Tables at the top right corners contain values of Spearman's rank correlation coefficient. Correlation coefficients significance level $P$ values marked as: * $P \leq 0.05 ; * * P \leq 0.01$; ** $P \leq 0.001$.

at inhibitory doses reduces transcription rate of mentioned genes and simultaneously increases transcriptional activity of AtRad1.

It is crucial that the stimulation or inhibition of growth, growth delay, appearance of phenotypic deviations (anomalies of habitus and development) in irradiated plants and in their progeny significantly correlate with the transcription level of the genes which have been studied. In particular, the survival and growth of irradiated plants are negatively correlated with the activity of gene AtRad1. Radiation-induced expression of AtKu70 and AtRAD51 has a stimulating effect on the growth and development of $A$. thaliana after fractionated radiation exposure. A suppression of AtKu70 combined with low transcriptional activity of AtRAD51 vice versa leads to decreased survival, inhibition of growth and development, formation of abnormal phenotype of 
plants in post-radiation period. Therefore, relative transcription rates of the genes AtRAD51, AtKu70 and AtRad1 affect growth and development of plants, exposed to X-ray radiation, as well as appearance of abnormal phenotype of some plants in $\mathrm{F}_{1}$ generation. Transcriptional repression of AtKu70 and simultaneously up-regulation of AtRad1, involved in the error-prone DSB DNA repair pathways MMEJ and SSA, seem to be associated with radiation-induced genome instability resulting in phenotype deviations, stem growth retardation and reduced number of flowers per plant. Additionally, the data show that radiation-induced delay of flowering phenotype apparently is determined by another molecular pathway than repair of the plant genome.

Thus, it is suggested that at least some of the radiation-induced effects, such as temporal stimulation of development, radioadaptation and genome instability could be partly explained by change of transcription rate and co-expression of caretaker genes AtKu70, AtRAD51 and AtRad1 (Fig. 3). Specific transcription factors, such as suppressor of gamma response 1 (SOG1) [2, 5], and RNA-interference can be considered as potential regulators of transcription of these genes.

\subsection{Co-regulation of Transcriptional Response of} Genes, Participating in Maintenance of the A. thaliana Genome Integrity

Obtained results demonstrate that mode and dose of irradiation significantly affect concentration of transcripts of caretaker genes AtKu70, AtRAD51 and AtRad 1 in cells of $A$. thaliana plants. The dependence of the transcription rate on dose is not linear. Moreover, there is reason to describe changes in transcription of genes AtRAD51 and AtKu70 under influence of X-ray radiation rather as regulation and modulation of expression than simply induction or suppression. It was found that cells in tissues of rosette leaves of 5-week-old Arabidopsis plants express gene AtRad1 constitutively and that this gene does not belong to the class of radiation-induced loci, such as AtRAD51. At the same time, gene AtKu70 is regulated in a complex constitutive-inducible manner. It was proposed that observed changes in transcription rate are based on two different, but complementary mechanisms: (1) "switch on/switch off" regulation and (2) "turn up/turn down" gene regulation. The first

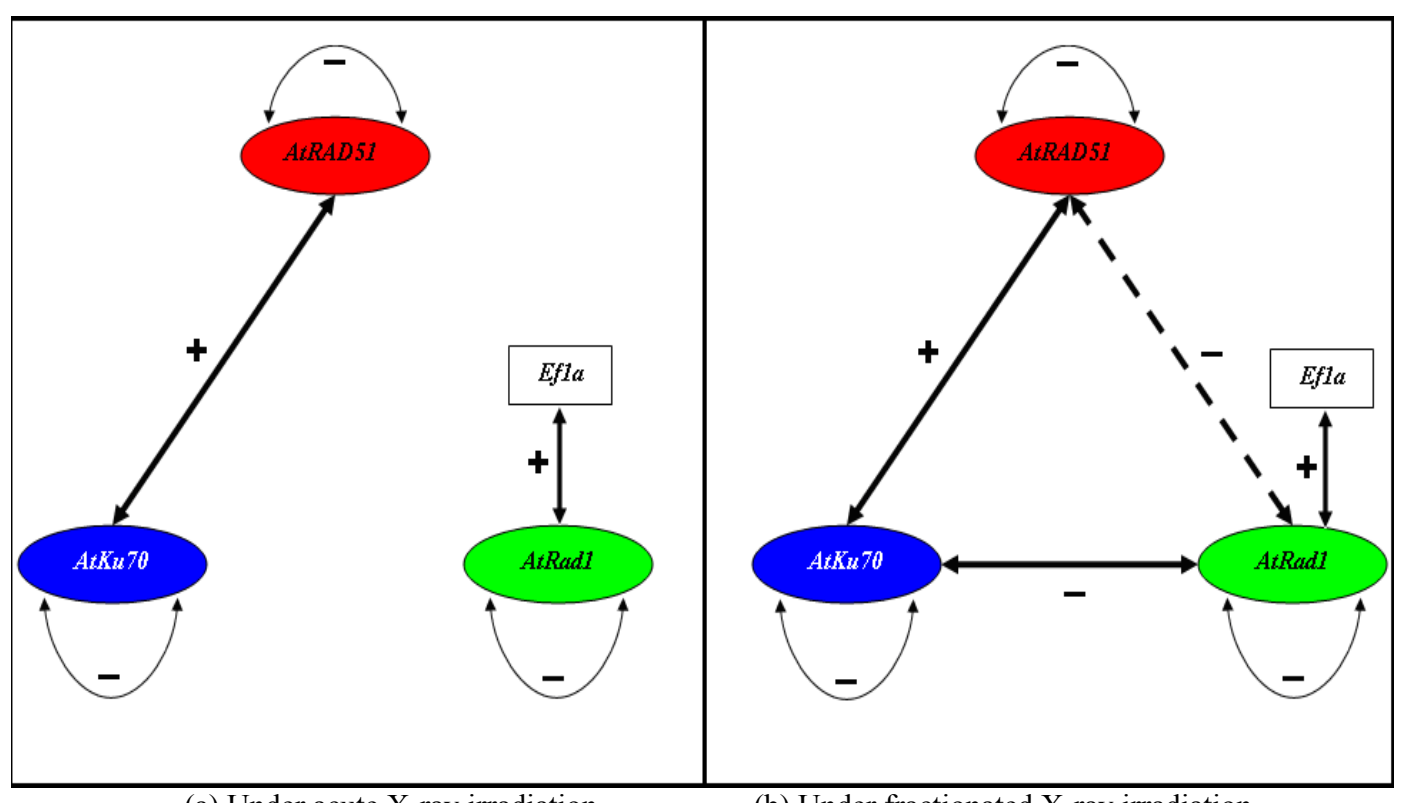

(a) Under acute X-ray irradiation

(b) Under fractionated X-ray irradiation

Fig. 3 Scheme of transcriptional co-regulation of AtKu70, AtRAD51 and AtRad1 under conditions of acute (a) and fractionated (b) X-ray irradiation. 
one is responsible for the general activation or repression of the gene, the second for a more fine-tuning or "quantitative" setting of its expression.

The wave-like character of the transcription activity-dose dependence curves might be explained by the model of negative feedback (Fig. 3). The solid bold arrows revealed significant Pearson partial correlation coefficients between the transcriptional activity of genes, which is a marker of possible co-regulation. The dotted line shows probably spurious correlation between AtRAD51 and AtRad1, caused by AtRad1-AtKu70 and AtRAD51-AtKu70 pair wise covariance (negative pair correlation coefficient is high, but partial correlation between transcription rates of genes AtRAD51 and AtRad1 is not statistically significant). The arc form arrow indicates the negative feedback.

Analysis of covariance of transcription level of mentioned repair genes under conditons of acute and fractionated radiation reveals to suggest down-regulation of AtRad1 by AtKu70 expression. It is likely that only mature $K u 70$ protein has a suppressive activity as a negative regulator of AtRad1, observed only under conditions of fractionated exposure. This can be explained by assuming that "turn down" of AtRad1 transcription takes more than $2 \mathrm{~h}$ after exposure for mRNA processing and translation of AtKu70. Negative regulation of AtRad1 by Ku70 protein is consistent with another data obtained for budding yeast and gives evidence of the conservativeness of this mechanism [13].

It should be emphasized that AtRAD51 and AtKu70 are obviously positively co-regulated. Under conditions of acute X-ray irradiation, the gene AtRad1 is regulated independently of AtKu70 and AtRAD51. Thus, it can be concluded that expression of inducible gene AtRAD51 and partly inducible gene AtKu70 is governed by the same transcriptional factor SOG1 [2, 5]. Regarding AtRad1, investigation confirms the regulation of its expression by SOG1-independent mechanism, as indicated by the observation that transcription activity of AtRad1 under X-ray exposure do not match by activity of the genes AtKu70 and AtRAD51.

\section{Conclusions}

The effect of X-ray radiation at different doses, significantly lower than median lethal dose for survival of $A$. thaliana plant, is mediated by the modification of expression of caretaker genes, participating in maintenance of the structural integrity of the cell's chromosomal apparatus. The positive or negative sign of the effect is determined by the ratio of activity of two groups of genes: those involved in the correct recovery of the structure of DNA and genes responsible for error-prone repair pathways.

X-ray radiation can lead to both radio adaptation response through the induction of transcription of AtRAD51 and AtKu70 genes, and genome instability through the transcriptional activation of error-prone AtRad1-mediated DNA DSB repair combined with decreased activity of high fidelity AtRAD51-depended repair. Overall, the transcriptional response of caretaker genes might play the main role in plant adaptation to the repeated exposure of genotoxic factors.

The obtained data allow to link phenotype deviations observed when doses of 3-21 Gy were used with a modification of the expression of key caretaker genes. Thus, an evidence in favor of the hypothesis about the impact of relatively small doses of radiation on the plant by a mechanism other than the direct induction of DNA damage has been received.

\section{Acknowledgments}

The authors wish to express their gratitude to the late academician Dmitry Mikhailovich Grodzinsky (Institute of Cell Biology and Genetic Engineering, National Academy of Sciences of Ukraine) for helpful comments and to scientific researcher Anatoly Pereguda (National Cancer Institute, Ukraine) for $\mathrm{X}$-ray exposure of plants. 


\section{References}

[1] Waterworth, W. M., Drury, G. E., Bray, C. M., and West, C. E. 2011. "Repairing Breaks in the Plant Genome: The Importance of Keeping It Together." New Phytologist 192 (4): 805-22.

[2] Yoshiyama, K. O., Sakaguchi, K., and Kimura, S. 2013. "DNA Damage Response in Plants: Conserved and Variable Response Compared to Animals." Biology 2 (4): 1338-56.

[3] Litvinov, S. 2014. "Effects of Chronic Exposure of Seeds and Seedlings of Arabidopsis thaliana by Low Doses of $\gamma$-Radiation on Plant Growth and Development." Nuclear Physics and Atomic Energy 15 (4): 406-14.

[4] Yokota, Y., Shikazono, N., Tanaka, A., Hase, Y., Funayama, T., Wada, S., and Inoue, M. 2005. "Comparative Radiation Tolerance Based on the Induction of DNA Double-Strand Breaks in Tobacco BY-2 Cells and CHO-K1 Cells Irradiated with Gamma Rays." Radiation Research 163 (5): 520-5.

[5] Mannuss, A., Trapp, O., and Puchta, H. 2012. "Gene Regulation in Response to DNA Damage." Biochimica et Biophysica Acta 1819 (2): 154-65.

[6] Christmann, M., and Kaina, B. 2013. "Transcriptional Regulation of Human DNA Repair Genes Following Genotoxic Stress: Trigger Mechanisms, Inducible Responses and Genotoxic Adaptation." Nucleic Acids Research 41 (18): 8403-20.

[7] Sidler, C., Li, D., Kovalchuk, O., and Kovalchuk, I. 2015. "Development-Dependent Expression of DNA Repair
Genes and Epigenetic Regulators in Arabidopsis Plants Exposed to Ionizing Radiation." Radiation Research 183 (2): 219-32.

[8] Bradford, W. D., Cahoon, L., Freel, S. R., Mays Hoopes, L. L., and Eckdahl, T. T. 2005. "An Inexpensive Gel Electrophoresis-Based Polymerase Chain Reaction Method for Quantifying mRNA Levels." Cell Biology Education 4 (2): 157-68.

[9] Hwang, J. Y., Smith, S., and Myung, K. 2005. "The Rad1-Rad10 Complex Promotes the Production of Gross Chromosomal Rearrangements from Spontaneous DNA Damage in Saccharomyces cerevisiae." Genetics 169 (4): 1927-37.

[10] McVey, M., and Lee, S. E. 2008. "MMEJ Repair of Double-Strand Breaks (Director's Cut): Deleted Sequences and Alternative Endings." Trends in Genetics 24 (11): 529-38.

[11] Scuric, Z., Chan, C.Y., Hafer, K., and Schiestl, R. H. 2009. "Ionizing Radiation Induces Microhomology-Mediated End Joining in Trans in Yeast and Mammalian Cells." Radiation Research 171 (4): 454-63.

[12] Li, J., Harper, L. C., Golubovskaya, I., Wang, C. R., Weber, D., Meeley, R. B., McElver, J., Bowen, B., Cande, W. Z., and Schnable, P. S. 2007. "Functional Analysis of Maize RAD51 in Meiosis and Double-Strand Break Repair." Genetics 176 (3): 1469-82.

[13] Decottignies, A. 2007. "Microhomology-Mediated End Joining in Fission Yeast Is Repressed by Pku70 and Relies on Genes Involved in Homologous Recombination." Genetics 176 (3): 1403-15. 